\title{
A VULNERABILIDADE DA FLORESTA AMAZÔNICA PERANTE AS MUDANÇAS CLIMÁTICAS
}

\author{
Philip M. Fearnside \\ Instituto Nacional de Pesquisas da Amazônia (INPA). C.P. 478. Manaus, Amazonas, Brasil. CEP: 69.011-970. \\ E-mail: pmfearn@inpa.gov.br
}

\section{RESUMO}

A floresta amazônica enfrenta sérias ameaças à sua sobrevivência devido às mudanças globais, que poderão tornar a Amazônia gradualmente mais quente e mais seca. Este efeito aparece mais intensamente em simulações do clima através de modelos que incluem a ligação entre o aquecimento da água no oceano Pacífico e a ocorrência do El Niño. Eventos como incêndios em Roraima em 1997/1998 e 2003 indicam que a ligação mencionada anteriormente é real. Os impactos são mais acentuados em modelos que incluem as retroalimentações bioesféricas, a morte da floresta e o aquecimento dos solos levando à emissão de carbono que, por sua vez, aumenta mais a temperatura e a morte da floresta. Uma ameaça climática inesperada revelouse em 2005, quando uma seca devastadora atingiu a Amazônia. Este tipo de seca se deve a um gradiente de temperatura da água da superfície do oceano entre o Atlântico Norte e Sul, que faz parte de uma oscilação que está intensificando. A formação da mancha de água quente no Atlântico Norte está se agravando devido à redução das cargas de aerossol sobre o mar nesta área, situação que deve se intensificar nas próximas décadas como resultado da continuação do aquecimento global. A concretização, de um cenário deste tipo depende de decisões humanas sobre a limitação das emissões de gases do efeito estufa, provenientes tanto da queima de combustíveis fósseis quanto do desmatamento. O Brasil é um dos países que perderia mais com o aquecimento global, colocando em risco a própria floresta amazônica. Portanto, o Brasil precisa mudar de lado nas negociações sob a Convenção de Clima. Ao invés de sempre tentar adiar a assunção de uma meta para redução de emissões, deve assumir imediatamente uma meta sob a Convenção (não apenas internamente), juntando ao Anexo I da Convenção e ao Anexo B do Protocolo de Kyoto, e passar a se empenhar para convencer os outros países, como a China e a Índia, a fazer o mesmo.

Palavras chave: Amazônia, aquecimento global, carbono, ciclagem d'água, desmatamento, efeito estufa, El Niño, redução de emissões.

\footnotetext{
ABSTRACT

THE VULNERABILITY OF THE AMAZON FOREST IN THE FACE OF CLIMATE CHANGE. The Amazon forest is threatened by ongoing climate changes, which are expected to make this region gradually hotter and drier. These effects are more intense in global climate simulations by models that connect peaks in the temperature of surface water in the Pacific Ocean with the El Niño phenomenon. Past events such as the fires in Roraima of 1997/1998 and 2003 suggest that the connection between the Pacific Ocean-surface temperature and El Niño is real. The estimated impact of climate changes on the Amazon are even worse in models that include biospheric feedback effects, pointing to the disappearance of forested areas and subsequent heating of the exposed soil, which leads to greater carbon emissions that further affect climate and kill more forest. A previously unappreciated climatic threat became apparent in 2005, when a devastating drought struck Amazonia. This type of drought is linked to a gradient of water temperature from the North to the South Atlantic Oceans, which is part of an oscillation that is becoming more intense. The formation of the patch of warm water in the tropical North Atlantic is worsening due to the reduction of aerosol loads over this area of
} 
the ocean, a situation that is expected to intensify in the forthcoming decades as a result of continued global warming. Whether such a scenario will come to take place depends on our efforts towards limiting the emission of greenhouse gases from fossil-fuel combustion and deforestation. Brazil is among the countries with the most to lose from global warming, possibly risking the loss of the Amazon rainforest. Therefore, Brazil ought to change political sides in negotiations under the Climate Convention. Instead of trying to postpone any commitment to reductions in its emissions, Brazil should immediately agree to a reduction target under the Convention (not only as an internal objective), thus joining the Convention's Annex I and Kyoto Protocol's Annex B, and then endeavor to convince other countries, such as China and India, to do likewise.

Keywords: Amazonia, global warming, carbon, water cycling, deforestation, greenhouse effect, El Niño, emissions reduction.

\section{RESUMEN}

LA VULNERABILIDAD DE LASELVAAMAZÓNICA FRENTE A LOS CAMBIOS CLIMÁTICOS.

La selva amazónica enfrenta serias amenazas para su sobrevivencia debido a los cambios globales, que se proyecta tornaran a la Amazonia más caliente y seca. Este efecto es mucho más intenso en simulaciones de clima global usando modelos que incluyen la conexión entre el calentamiento del agua en el océano Pacífico y la ocurrencia del fenómeno El Niño. Eventos como los incendios en Roraima en 1997/1998 y 2003 indican que la conexión con El Niño es real. Los impactos son peores en modelos que incluyen las retroalimentaciones bioesféricas, con la muerte del bosque y el calentamiento de los suelos llevando a la emisión de carbono que, a su vez, calienta más el clima y mata más bosque. Una amenaza climática que antes no era apreciada se reveló en 2005, cuando una sequia devastadora afectó a la Amazonia. Este tipo de sequia se debe a un gradiente de temperatura del agua de la superficie del mar entre el Atlántico Norte y el Atlántico Sur, que hace parte de una oscilación que se está intensificando. La formación de la mancha de agua caliente en el Atlántico Norte se está agravando debido a la reducción de las cargas de aerosol sobre el mar en esta área, situación que debe intensificarse en las próximas décadas como resultado de la continuación del calentamiento global. La concretización, o no, de un escenario de este tipo depende de decisiones humanas sobre la limitación de las emisiones de gases de efecto invernadero, tanto de la quema de combustibles fósiles como de la continuación de la deforestación. Brasil es uno de los países que perdería mas con el calentamiento global, con la propia selva amazónica estando en riesgo. Por lo tanto, Brasil debe cambiar de lado en las negociaciones bajo la Convención del Clima. Al contrario de siempre intentar posponer el compromiso con una meta para reducir sus emisiones, Brasil debe asumir inmediatamente una meta frente a la Convención (no solo de forma interna), adhiriéndose al Anexo I de la Convención y al Anexo B del Protocolo de Kyoto, sino pasando a impulsar y convencer a los otros países, como China e India, a hacer lo mismo.

Palabras clave: Amazonía, calentamiento global, carbono, ciclo del agua, deforestación, efecto invernadero, El Niño, reducción de emisiones.

\section{PREDIÇÕES DE MODELOS DE CLIMA}

Diferentes modelos climáticos produzem uma gama extensiva de resultados para o clima futuro na Amazônia. Um modelo em particular, o modelo do Centro Hadley do Centro de Meteorologia do Reino Unido, indica uma mudança catastrófica para um clima mais seco e quente na Amazônia, resultando na morte de quase toda a floresta até 2080 (Cox et al. 2000, 2004). Na época do Quarto Relatório de Avaliação (AR-4) do Painel Intergovernamental sobre Mudanças Climáticas (IPCC), de 2007, vários outros modelos indicavam a Amazônia se tornando significativamente mais seca, dentre estes o modelo do Centro Nacional de Pesquisas Atmosféricas (NCAR), dos EUA, e o modelo ECHAM do Instituto Max Planck, da Alemanha. Alguns modelos, como o do CSIRO, da Austrália, não indicavam nenhuma mudança na Amazônia, enquanto um modelo, do Laboratório Geofísico de Dinâmica de Fluidos (GFDL), nos E.U.A., indicava mais chuva na Amazônia (veja Kundzewicz et al. 2007, pág. 183). A indicação de aumento de chuva na Amazônia no modelo do GFDL era o resultado de um erro no modelo que já foi 
corrigido (S.M. Griffies, comunicação pessoal, 2009). Mesmo assim, os resultados são bastante variados, e é importante avaliar os modelos diferentes para o propósito específico de representar o clima futuro na Amazônia, assim como também considerar a melhor maneira de interpretar o significado da incerteza restante para a política.

Os resultados catastróficos do modelo do Centro Hadley foram publicados pela primeira vez na revista Nature em 2000. É extremamente inquietante que nove anos de trabalho intensivo de vários grupos de pesquisa não tenha identificado um erro específico que invalidaria este resultado, embora os resultados dos outros modelos sejam comparativamente menos catastróficos. Um pouco de conforto para nós deriva do fato de que o modelo Hadley indica um clima atual na Amazônia que é mais quente e mais seco do que é o real clima de hoje (Cândido et al. 2007). Isto significa que, provavelmente, são exagerados também os valores numéricos para temperatura e seca no clima futuro simulado. Porém, o clima futuro simulado vai tão além dos limites de tolerância de árvores da floresta amazônica que causaria uma grande mortalidade até mesmo se as mudanças forem menos extremas do que as simulações indicam.

\section{EL NIÑO E O EFEITO ESTUFA}

A pergunta central no que se refere aos resultados do modelo do Centro Hadley para a Amazônia é se a sua representação dos efeitos do fenômeno El Niño está correta. Nesse modelo, a continuação do aquecimento global leva o sistema climático a permanecer em um "El Niño permanente", resultando em severas secas e calor na Amazônia. Só parte desta seqüência é mostrada por outros modelos.

O Painel Intergovernamental sobre Mudanças Climáticas (IPCC) observou no seu Segundo Relatório de Avaliação, de 1995, que a freqüência de eventos El Niño foi muito mais alta desde 1976 em comparação com os anos anteriores, uma diferença estatística altamente significativa (Nicholls et al. 1996, pág. 165). Eventos recentes, como os El Niños de 1997 e 2003, tiveram importantes impactos na Amazônia.

O Quarto Relatório de Avaliação do IPCC (AR-4), de 2007, conclui que a continuação do aquecimento global conduziria à formação de "condições tipo El Niño" (Meehl et al. 2007, pág. 779). Isto se refere às águas superficiais mais mornas no Oceano Pacífico, que é o gatilho para o El Niño. Porém, o relatório do IPCC observa que os vários modelos de clima ainda não concordam em uma ligação entre o aquecimento global e o El Niño em si (Meehl et al. 2007, pág. 780). Isto refere às secas e inundações em locais diferentes ao redor do mundo.

Infelizmente, nós sabemos de forma direta que condições tipo El Niño conduzem à seca e incêndios florestais na Amazônia; esta não é uma conclusão que depende dos resultados de modelos de clima. As secas do El Niño em 1982, 1997 e 2003 fornecem exemplos que são lembrados pela maioria das pessoas na Amazônia. O gráfico das temperaturas da superfície do mar no Oceano Pacífico (Hansen et al. 2006; McPhaden et al. 2006) é um retrato perfeito de eventos de seca na Amazônia. A ilustração usada por Al Gore no seu filme "Uma Verdade Inconveniente" é altamente pertinente. Da mesma maneira que os continentes da África e da América do Sul se ajustam perfeitamente demais para se tratar de uma coincidência, os gráficos de $\mathrm{CO}_{2}$ atmosférico e de temperatura global se espelham um no outro tão bem que deve haver uma relação entre os dois. $\mathrm{O}$ mesmo se aplica para temperatura de superfície do mar no Pacífico e secas amazônicas. Isto significa que uma tabulação simples dos resultados de diferentes modelos de clima não é suficiente. Se um modelo mostra a água superficial esquentando no Pacífico, mas nada acontecendo na Amazônia, então significa que há algo faltando no modelo, não que nós estamos mais seguros na Amazônia.

O El Niño é um fenômeno que é difícil de representar em modelos de circulação global (GCMs), em parte por causa da resolução espacial grosseira que é ditada pela capacidade de processamento da maioria dos supercomputadores de hoje. Porém, é inquietante que o Simulador da Terra, um enorme complexo de computadores em Yokohama, Japão, também produza resultados catastróficos quando programado com uma física de clima semelhante à do modelo do Centro Hadley. Picos de temperaturas na Amazônia central de mais de $50^{\circ} \mathrm{C}$ se tornariam comuns a partir de 2050 em cenários business-asusual. O Simulador da Terra representa o planeta em células de grade ("pixels") de $10 \times 10 \mathrm{~km}$, enquanto outros computadores que executam GCMs usam células de grade de aproximadamente $300 \times 300 \mathrm{~km}$. 
O El Niño produz um padrão de inundações e secas ao redor do mundo, com pesadas chuvas na costa do Peru, seca na parte norte da Amazônia (por exemplo, o Grande Incêndio de Roraima de 1997-1998), inundações em Santa Catarina, seca no Bornéu (que também provocou incêndios em 1997-1998), seca na Etiópia (que matou mais de 200.000 pessoas em 1982) e calor na Europa (que matou aproximadamente 40.000 pessoas em 2003). Conseguir um modelo de clima para representar todos estes efeitos simultaneamente quando a água do Pacífico esquenta é uma tarefa difícil e, esta dificuldade explica porque os modelos diferentes atualmente não concordam entre si. Porém, do ponto de vista de seca amazônica, nós apenas precisamos representar esta parte do padrão global corretamente, e não o resultado em todos os outros locais que também são afetados pelo El Niño. Nisto, o modelo do Centro Hadley fez o melhor reproduzindo a conexão entre água morna no Pacífico (i.e., "condições tipo El Niño") e secas amazônicas. Entre 21 modelos testados para esta habilidade pelo Projeto em Conjunto de Inter-comparação de Modelos (CMIP2), o modelo do Centro Hadley foi classificado em primeiro lugar (veja Cox et al. 2004).

\section{OSCILAÇÃO ATLÂNTICA}

Uma ameaça climática que antes não era apreciada revelou-se em 2005, quando uma seca devastadora atingiu a Amazônia. As vazões nos afluentes do lado sul do Rio Amazonas foram tão baixas que embarcações não puderam navegar nos rios e comunidades ribeirinhas ficaram isoladas de hospitais e outros serviços essenciais. Incêndios florestais queimaram no Acre e em partes vizinhas do Estado de Amazonas, um evento sem precedentes (Brown et al. 2006; Vasconcelos \& Brown, 2007). A floresta perdeu biomassa por causa da diminuição do crescimento e do aumento da mortalidade de árvores (Phillips et al. 2009). O ano de 2005 não foi um ano de El Niño: ao invés de água mais quente do que o normal no Pacífico, havia água quente na parte sul do Atlântico Norte e água fria na parte norte do Atlântico Sul. A água quente no Atlântico Norte deu energia ao Furacão Katrina, que atingiu a cidade de Nova Orleans nesse ano. Também contribuiu para a seca na Amazônia por causar maior ascenção de ar quente quando a Zona de Convergência Intertropical
(ITCZ) atingiu a área de água quente. O ar no ITCZ sobe até uma altitude de aproximadamente $1.800 \mathrm{~m}$, se divide em fluxos para o norte e para o sul, e então se move no sentido dos pólos por aproximadamente $30^{\circ}$ de latitude antes de descender ao nível do solo e retornar para o Equador em baixa altitude, assim formando a célula de Hadley. Quando o ar sobe, seu teor de umidade condensa e cai como chuva, e depois, quando o ar desce ao nível do solo, é seco, e resseca a área atingida pelo ar descendente. Com mais ar quente e úmido subindo na ITCZ em 2005, houve também mais ar frio e seco descendo $30^{\circ}$ mais ao Sul. Com o gradiente de temperatura entre a água quente no Atlântico Norte e fria no Atlântico Sul, a ITCZ foi puxada mais ao Norte do que o habitual e, na época do ano quando o movimento sazonal do ITCZ foi perto de seu extremo Norte, o ar seco estava descendo sobre as nascentes dos afluentes do rio Amazonas, do lado sul da bacia (e.g., Fearnside, 2006a; Marengo et al. 2008).

A água quente do Atlântico Norte em 2005 foi o resultado combinado de vários fatores. Um era a Oscilação Multi-decadal do Atlântico (AMO), que produz água mais quente do que a média nesta área em intervalos de aproximadamente 40 anos. No entanto, esta oscilação explica, no máximo, $11 \%$ da anomalia de temperatura em 2005 , enquanto $50 \%$ do aumento da temperatura poderiam ser diretamente atribuídos ao aquecimento global (Trenberth \& Shea, 2006). Houve também uma contribuição indiretamente ligada ao aquecimento global, de $22 \%$, proveniente de vestígios de um período de El Niño nos anos anteriores. Além disso, a redução da carga de aerossóis atmosféricos sobre o Atlântico resultou em menos proteção desta área de oceano contra a radiação solar. A carga de aerossóis está diminuindo tanto devido à redução da poluição atmosférica industrial na Europa e América do Norte (Cox et al. 2008) como devido à redução da carga de poeira oriunda da África (Evan et al. 2009). As cargas reduzidas de aerossol são responsáveis por $69 \%$ da tendência ascendente na temperatura da superfície do mar nesta região entre 1985 e 2005, período em que a temperatura da água aumentou em $0,6^{\circ} \mathrm{C}$ (Evan et al. 2009). Esta redução de aerossol é consistente com resultados modelados de aquecimento global, que indicam que a duplicação do $\mathrm{CO}_{2}$ atmosférico pré-industrial (prevista para ocorrer 2070, ou antes, presumindo emissões nos 
níveis de business-as-usual) reduziria a cobertura de poeira sobre o Atlântico em $40-60 \%$ e aumentaria a temperaturas da superfície do mar em 0,3 a $0,4^{\circ} \mathrm{C}$ adicionais (Mahowald \& Luo 2003).

O gradiente Norte-Sul de temperatura no Atlântico está significativamente correlacionado com as chuvas na porção Sudoeste da Amazônia e, em 2005, tanto o gradiente no Atlântico como a seca no sudoeste da Amazônia chegaram até níveis extremos (Cox et al. 2008). Resultados do modelo do Centro Hadley indicam um aumento enorme na amplitude do gradiente de temperatura no Atlântico e nas secas associadas na Amazônia, se as emissões de gases de efeito estufa continuarem seu caminho atual (Cox et al. 2008). Os resultados de simulação indicam que a probabilidade de uma seca tão grave como a de 2005 foi de 5\% (1 ano em 20) em 2005, mas isso aumentaria para 50\% (1 ano em 2) até 2025 e 90\% (9 anos em 10) até 2060. A probabilidade de ocorrência dessas secas explode se o teor atmosférico de $\mathrm{CO}_{2}$ for superior a 400 ppmv, um nível apenas ligeiramente superior ao nível de 2009 de 387 ppmv. As concentrações atmosféricas de $\mathrm{CO}_{2}$ estão aumentando em cerca de 2,4 ppmv ao ano.

\section{SAVANIZAÇÃO DA AMAZÔNIA}

A perda de grandes áreas de floresta amazônica devido às mudanças climáticas não depende do modelo do Centro Hadley ser a melhor representação do clima futuro. Salazar et al. (2007) testaram 15 modelos diferentes para as implicações para a "savanização" na Amazônia. Mais de 75\% dos modelos indicam que uma faixa que hoje é floresta ao longo das extremidades leste e sul da região será climaticamente imprópria para floresta até 2100 , levando à substituição de árvores por outro tipo de vegetação, geralmente denominado como "savana". Pelo menos $25 \%$ dos modelos indicam uma mudança deste tipo em toda a parte da região amazônica a leste de Manaus. O modelo do Centro Hadley, é claro, mostraria que a savanização ocorreria em toda a floresta amazônica brasileira neste prazo.

Os vários modelos de clima, inclusive o modelo do Centro Hadley, omitem vários processos críticos que podem fazer com que os eventos reais sejam até mais desastrosos do que aqueles indicados pelos modelos. Os modelos só mostram os efeitos do aquecimento global, mas a floresta amazônica está sujeita a outros fatores de estresse. O mais óbvio é o desmatamento direto, com derrubada de árvores por motosserras em lugar de morte por falta d'água. Isto não só elimina as árvores que são derrubadas diretamente, mas também contribui com as outras mudanças climáticas que reforçam as mesmas tendências ao clima mais quente e mais seco para o resto da floresta, assim contribuindo com a morte da floresta como um todo. A perda de árvores reduz a evapotranspiração, reduzindo a chuva sobre o resto da floresta (e.g., Lean et al. 1996). Duas recentes simulações indicam que a perda contínua de floresta conduziria a clima mais quente e mais seco no resto da região (Foley et al. 2007, Sampaio et al. 2007). Se o desmatamento seguir os padrões espaciais projetados, uma queda abrupta da quantidade de chuva na estação seca ocorreria depois que o desmatamento alcançar 40\% (Sampaio et al. 2007). Até 2008, o desmatamento havia removido $18,4 \%$ da floresta original na Amazônia brasileira (Brasil, INPE 2009). A estação seca é o período crítico do ano quando árvores podem morrer por falta d'água.

Incêndios florestais representam uma grande ameaça para florestas amazônicas e, são omitidos dos GCMs como o modelo do Centro Hadley. Especialmente em anos El-Niño, fogos podem se mover pelo sub-bosque da floresta, matando grandes árvores. No Grande Incêndio de Roraima de 19971998, uma área calculada em 11-13.000 $\mathrm{km}^{2}$ de floresta foi queimada (Barbosa \& Fearnside 1999). Áreas grandes de floresta também queimaram no Pará (Alencar et al. 2004, 2006; Cochrane et al. 1999). As árvores mortas pelos fogos fornecem combustível para fogos subseqüentes, assim conduzindo a processos de retroalimentação positiva que destroem a floresta completamente ao longo de um período de vários anos (e.g., Nepstad et al. 2001). Uma vez que pode ser esperado que a mudança de clima aumente a freqüência e a severidade de incêndios, a floresta poderia ser morta mais depressa do que os modelos indicam. Um estudo recente indica perda significativa de floresta por incêndios antes de 2030 sob a suposição otimista de que os padrões de clima dos últimos 10 anos continuem inalterados (Nepstad et al. 2007). Os atuais padrões de variação climática na Amazônia implicam em risco para grandes áreas de floresta (Hutyra et al. 2005, Nepstad et al. 2004).

$\mathrm{O}$ modelo do Centro Hadley foi o primeiro a 
incluir "retroalimentações bióticas", onde o carbono liberado da mortalidade de floresta e do aquecimento do solo é incluído no calculo do efeito estufa futuro, que, em troca, conduz a maior liberação de carbono biosférico terrestre. Com o modelo do Centro Hadley, a temperatura média global em 2100 é 38\% mais alta se as retroalimentações bióticas são incluídas. Porque apenas aproximadamente um quinto dos 20 modelos usados pelo AR-4 do IPCC tiveram a capacidade para incluir retroalimentações bióticas, essa parte de todos os modelos foi desativada nas execuções usadas para as estimativas do IPCC da temperatura global (i.e., o aumento de $4^{\circ} \mathrm{C}$ em cima da temperatura pré-industrial até 2100 sob o cenário "A-2" que melhor aproxima as tendências atuais). Com certeza, as avaliações futuras do IPCC incorporarão estas retroalimentações, nas quais a Amazônia desempenha um papel principal.

\section{RISCO E INCERTEZA}

As predições de clima futuro indicam risco considerável para a Amazônia, assim como uma incerteza significativa. A maneira em que estes dois fatores estão incorporados em decisões sobre políticas públicas pode fazer uma grande diferença nas ações tomadas e, conseqüentemente na sobrevivência da floresta amazônica.

Incerteza se refere à falta de conhecimento sobre valores dos parâmetros ou sobre quais são as verdadeiras probabilidades de cada resultado diferente acontecer. A existência de incerteza tem sido usada repetidamente para evitar a tomada de difíceis decisões sobre a mudança climática. $\mathrm{O}$ caso mais notório é a recusa tradicional do presidente dos EUA, George W. Bush, em reconhecer a existência do efeito estufa, assim justificando a sua recusa de assumir compromissos específicos para reduzir as emissões. O Brasil adotou uma posição semelhante quando o relatório do IPCC sobre os impactos de mudança do clima foi aprovado em Bruxelas em abril de 2007, fazendo uma tentativa mal sucedida para retirar do resumo do relatório para formuladores de política a menção do risco de savanização na Amazônia (Folha de São Paulo 2007). Enquanto os governos recusam admitir a existência de um problema não há nenhuma necessidade de ações sérias para evitar o problema.

No caso da contribuição do Brasil para a emissão de gases de efeito estufa por meio de desmatamento, houve uma longa história de estimativas oficiais que suavizam ou minimizam a magnitude e importância deste fator (veja Fearnside 1997, 2000) A incerteza é freqüentemente invocada para justificar omissões. Por exemplo, a estimativa oficial das emissões do Brasil na Comunicação Nacional submetida à Convenção Quadro das Nações Unidas sobre Mudanças de Clima (UN-FCCC) em dezembro de 2004 não contabilizou o carbono nas raízes das árvores por razões de incerteza (Brasil, MCT, 2004, pág. 148). Isto por si só aumentaria as emissões em aproximadamente 20\%, no caso de floresta Amazônica, e mais do que o dobro no caso de cerrado. Uma série de fatores ou são omitidos completamente ou são representados por valores otimistas improváveis (ver Fearnside \& Laurance 2004).

A maior parte daquilo que é discutido com relação às mudanças climáticas, inclusive nos relatórios do IPCC, é exclusivamente baseado em médias ou estimativas "mais prováveis". O caso "mais provável" significa que há uma probabilidade de 50\% que o valor real seja igual ou menor que a estimativa. Porém, o outro lado desta moeda é que há uma chance de $50 \%$ de que o valor real seja mais alto, e talvez muito mais alto. $\mathrm{O}$ que deveria ser a resposta para esta incerteza em termos de política? O princípio de precaução indicaria que políticas deveriam ser conservadoras para assegurar que limiares críticos não seriam ultrapassados. Em outras palavras, menos desmatamento deveria ser permitido e as emissões globais de gases de efeito estufa deveriam ser limitadas em níveis mais baixos do que aqueles que seriam indicados por modelos usando valores "médios" ou "mais prováveis".

Além da incerteza associada ao clima na Amazônia, há incerteza considerável relativa ao sistema climático global. Um fator chave que determina a severidade dos impactos do aquecimento global é a "sensitividade climática", ou a quantia pela qual a temperatura média global em equilíbrio aumentaria como resultado de uma duplicação da concentração de $\mathrm{CO}_{2}$ pré-industrial de 280ppmv. Sob cenários do tipo business-as-usual esta duplicação ocorre até 2070. O "provável" valor para sensitividade climática é aproximadamente $3^{\circ} \mathrm{C}$, mas há uma chance de $50 \%$ que o verdadeiro valor seja mais alto que isto e a possibilidade de que é muito mais alto é significante. Um valor de $6,2^{\circ} \mathrm{C}$ precisaria ser usado para ter $95 \%$ de certeza que o valor real 
é incluído (Hegerl et al. 2006). A projeção de uma elevação de $4^{\circ} \mathrm{C}$ da temperatura média global sobre os níveis pré-industriais até 2100 é baseada em uma sensitividade climática de cerca de $3^{\circ} \mathrm{C}$. Isto também é verdade para as várias simulações de savanização na Amazônia.

O perigo de se usar valores médios ou "mais prováveis" nas decisões sobre eventos catastróficos pode ser ilustrado por um exemplo simples. Imagine que alguém que mora em um prédio de apartamentos fosse perguntar para um engenheiro se o edifício desmoronará e cairá ao chão como o Edifício Palace II, que desmoronou no Rio de Janeiro em 1998. Se o engenheiro respondesse que é "provável” que o edifício continue de pé, o residente preocupado ficaria satisfeito? Claramente a resposta é "não", já que pode ter, por exemplo, uma chance de $51 \%$ que o edifício continuar de pé, porém, uma chance de $49 \%$ que se desmorone! Seguramente uma pessoa que mora no edifício, para quem um colapso seria catastrófico, necessitaria de uma probabilidade muito maior do que 99\% de que o edifício permanece de pé. Quanto mais catastrófico o resultado, mais garantia é requerida que a catástrofe não acontecerá. Eventos como a morte da floresta amazônica seriam catastróficos para o Brasil, e, portanto, o Brasil deveria estar exigindo cortes mais fundos nas emissões globais totais. Porém, ao invés disso, a posição do Brasil foi uma recusa durante anos para especificar um limite sobre as emissões globais.

A UN-FCCC, assinada em 1992 no Rio de Janeiro no ECO-92 tem como objetivo a estabilização de concentrações atmosféricas de gases de efeito estufa em níveis que evitariam interferência "perigosa" com o sistema climático global (UN-FCCC 1992, Artigo 2). Negociações estão em curso para definir o que seria "perigoso" em termos de uma concentração máxima de gases de efeito estufa equivalente a $\mathrm{CO}_{2}$ ou um valor máximo correspondente de elevação da temperatura global. Em março de 2005, a União Européia adotou $2^{\circ} \mathrm{C}$ de aumento da temperatura global sobre a média pré-industrial como a definição de "perigoso". Isto corresponde aproximadamente aos limites de tolerância da floresta amazônica. Somente em julho de 2009 o Presidente Luiz Inácio Lula da Silva endossou este alvo geral durante a reunião do G-8 em Áquila, Itália. Por que, então, o Brasil esperou até que mais de 100 países tivessem endossado este limite? A resposta, evidentemente, é que adotar um limite significa que todos os países, inclusive o Brasil, terão que fazer reais reduções nas suas emissões. Um limite global em termos de concentração ou em termos de temperatura significa que todas as emissões têm que ficar dentro do limite, independentemente de se elas são antropogênicas ou naturais, se elas são intencionais ou acidentais, e se elas vêm de países ricos ou pobres.

O Brasil deveria estar na liderança em fazer compromissos para reduzir as emissões porque é um dos países pesadamente afetados por mudanças climáticas projetadas e por causa de sua posição praticamente única de ter o grosso das suas emissões vindo de desmatamento, que contribui pouco à economia. Ao invés disso, o Ministério das Relações Exteriores do Brasil tem constantemente se recusado a assumir qualquer compromisso internacional para reduzir as emissões. Tradicionalmente tem até mesmo se recusado a fixar metas internas para redução das emissões, mas em 2009 o Itamaraty admitiu que, dentro de em breve, admitirá "ações quantificadas" na redução das emissões (Folha de São Paulo 2009). Embora rotuladas como "metas" pela imprensa, não se referem a compromissos internacionais sob a Convenção de Clima, nem a "metas" que impliquem em conseqüências caso não sejam cumpridas. As afirmações repetidas do governo de que o desmatamento está sob controle deveriam conduzir ao governo ser disposto a assumir compromissos para reduzir o desmatamento e as suas emissões associadas. Embora o desmatamento esteja menos "sob controle" do que implica a queda na taxa de desmatamento em $50 \%$ entre 2004 e 2007 , muito poderia ser feito para reduzir o desmatamento se fosse dado ao problema a prioridade que ele merece (e.g., Fearnside 2005). O custo de tais ações poderia ser compensado facilmente pelo valor das emissões que seriam evitadas se o desmatamento fosse reduzido e se o Brasil vendesse créditos de carbono dessa fonte que fossem válidos para compromissos internacionais (e.g., Fearnside 2006b). As contribuições brasileiras à Conferência das Partes (COP) da UN-FCCC em Nairobi em 2006 e em Bali em 2007 pelo menos abriram as portas à discussão deste assunto previamente considerado um tabu. Os governadores dos nove estados da Amazônia Legal brasileira já pediram em carta ao Presidente da República, que a posição brasileira seja mudada para "inclusão das florestas no mercado de carbono 
regulado por Kyoto" (O Estado de São Paulo 2009). Devido ao sistema climático levar décadas para responder às reduções de emissões, não há tempo a desperdiçar se é para conter a ameaça à floresta amazônica devido à mudança de clima.

AGRADECIMENTOS: Ao Conselho Nacional do Desenvolvimento Científico e Tecnológico (CNPq: Proc. 305880/2007-1), Rede GEOMA e Instituto Nacional de Pesquisas da Amazônia (INPA: PRJ13.03) pelo apoio financeiro. Agradeço também aos dois referees anônimos pelos comentários.

\section{REFERÊNCIAS}

ALENCAR, A.; NEPSTAD, D.C. \& VERA DIAZ, M. del C. 2006. Forest understory fire in the Brazilian Amazon in ENSO and nonENSO years: area burned and committed carbon emissions. Earth Interactions, 10(6): 1-17.

ALENCAR, A.C.; SOLÓRZANO, L.A. \& NEPSTAD, D.C. 2004. Modeling forest understory fires in an eastern Amazonian landscape. Ecological Applications, 14(4): S139-S149.

BARBOSA, R.I. \& FEARNSIDE, P.M. 1999. Incêndios na Amazônia brasileira: Estimativa da emissão de gases do efeito estufa pela queima de diferentes ecossistemas de Roraima na passagem do evento "El Niño" (1997/98). Acta Amazonica, 29(4): 513-534.

BRASIL, INPE (Instituto Nacional de Pesquisas Espaciais), 2009. Projeto PRODES: Monitoramento da Floresta Amazônica Brasileira por Satélite. INPE, São José dos Campos, São Paulo, Brasil. (Disponível em: http://www.obt.inpe.br/prodes/).

BRASIL, MCT (Ministério da Ciência e Tecnologia), 2004. Brazil's Initial National Communication to the United Nations Framework Convention on Climate Change. Ministry of Science and Technology (MCT), Brasília, DF, Brasil, 271p.

BROWN, I.F.; SCHROEDER, W.; SETZER, A.; MALDONADO, M.J.R.; PANTOJA, N.; DUARTE, A.F. \& MARENGO, J. 2006. Monitoring fires in Southwestern Amazonia rain forest. EOS, Transactions of the American Geophysical Union, 87(26): 253264.

CÂNDIDO, L.A.; MANZI, A.O.; TOTA, J.; DA SILVA, P.R.T.; DA SILVA, F.S.M.; dos SANTOS, R.N.N. \& CORREIA, F.W.S. 2007. O Clima atual e futuro da Amazônia nos Cenários do IPCC: A questão da savanização. Ciência e Cultura, 59(3): 44-47.
COCHRANE, M.A.; ALENCAR, A.; SCHULZE, M.D.; SOUZA JR., C.M.; NEPSTAD, D.C.; LEFEBVRE, P. \& DAVIDSON, E.A. 1999. Positive feedbacks in the fire dynamic of closed canopy tropical forests. Science, 284: 1832-1835.

COX, P.M.; BETTS, R.A.; COLLINS, M.; HARRIS, P.P.; HUNTINGFORD, C. \& JONES, C.D. 2004. Amazonian forest dieback under climate-carbon cycle projections for the $21 \mathrm{st}$ century. Theoretical and Applied Climatology, 78: 137-156, doi: 10.1007/s00704-004-0049-4.

COX, P.M.; BETTS, R.A.; JONES, C.D.; SPALL, S.A. \& TOTTERDELL, I.J. 2000. Acceleration of global warming due to carbon-cycle feedbacks in a coupled climate model. Nature, 408: 184-187.

COX, P.M.; HARRIS, P.P.; HUNTINGFORD, C.; BETTS, R.A.; COLLINS, M.; JONES, C.D.; JUPP, T.E.; MARENGO, J.A. \& NOBRE, C.A. 2008. Increasing risk of Amazonian drought due to decreasing aerosol pollution. Nature, 453: 212-215.

EVAN, A.T.; VIMONT, D.J.; HEIDINGER, A.K.; KOSSIN; J.P. \& BENNARTZ, R. 2009. The role of aerosols in the evolution of tropical North Atlantic ocean temperature anomalies. Science, 324: 778-781.

FEARNSIDE, P.M. 1997. Monitoring needs to transform Amazonian forest maintenance into a global warming mitigation option. Mitigation and Adaptation Strategies for Global Change, 2(2-3): 285-302.

FEARNSIDE, P.M. 2000. Effects of land use and forest management on the carbon cycle in the Brazilian Amazon. Journal of Sustainable Forestry, 12(1-2): 79-97.

FEARNSIDE, P.M. 2005. Deforestation in Brazilian Amazonia: History, rates and consequences. Conservation Biology, 19(3): 680-688.

FEARNSIDE, P.M. 2006a. A vazante na Amazônia e o aquecimento global. Ciência Hoje, 38(231): 76-78.

FEARNSIDE, P.M. 2006b. Mitigation of climatic change in the Amazon. p. 353-375. In: W.F Laurance \& C.A. Peres, (eds.), Emerging Threats to Tropical Forests. University of Chicago Press, Chicago, Illinois, E.U.A. 563p.

FEARNSIDE, P.M. \& LAURANCE, W.F. 2004. Tropical deforestation and greenhouse gas emissions. Ecological Applications, 14(4): 982-986.

FOLEY, J.A.; ASNER, G.P.; COSTA, M.H.; COE, M.T.; DEFRIES, R.; GIBBS, H.K.; HOWARD, E.A.; OLSON, S.; PATZ, J.; RAMANKUTTY, N. \& SNYDER, P. 2007. Amazonia revealed: forest degradation and loss of ecosystem goods and 
services in the Amazon Basin. Frontiers in Ecology and the Environment, 5(1): 25-32.

FOLHA DE SÃO PAULO. 2007. "Conclusão de texto involve debate intenso", 06 de abril de 2007, p. A-14.

FOLHA DE SÃO PAULO. 2009. "Itamaraty confirma que Brasil terá meta contra aquecimento", 12 de agosto de 2009, p .A-16.

HEGERL, G.C.; CROWLEY, T.J.; HYDE, W.T. \& FRAME, D.J. 2006. Climate sensitivity constrained by temperature reconstructions over the past seven centuries. Nature, 440: 10291032.

HANSEN, J.; SATO, M.; RUEDY, R.; LEA, D.W. \& MEDINAELIZADE, M. 2006. Global temperature change. Proceedings of the National Academy of Sciences, 203(39): 14288-14293.

HUTYRA, L.R.; MUNGER, J.W.; NOBRE, C.A.; SALESKA, S.R.; VIEIRA, S.A. \& WOFSY, S.C. 2005. Climatic variability and vegetation vulnerability in Amazonia, Geophysical Research Letters, 32, L24712, doi: 10.1029/2005GL024981.

KUNDZEWICZ, Z.W.; MATA, L.J.; ARNELL, N.W.; DÖLL, P.; KABAT, P.; JIMÉNEZ, B.; MILLER, K.A; OKI, T.; SEN, Z. \& SHIKLOMANOV, I.A. 2007. Freshwater resources and their management. p. 173-210 In: Parry, M.L.; Canziani, O.F.; Palutikof, J.P.; van der Linden, P.J.; Hanson, C.E. (eds.) Climate Change 2007: Impacts, Adaptation and Vulnerability. Contribution of Working Group II to the Fourth Assessment Report of the Intergovernmental Panel on Climate Change, Cambridge University Press, Cambridge, Reino Unido, 976p.

LEAN, J.; BUNTON, C.B.; NOBRE, C.A. \& ROWNTREE, P.R. 1996. The simulated impact of Amazonian deforestation on climate using measured ABRACOS vegetation characteristics. p. 549-576. In: J.H.C. Gash, C.A. Nobre, J.M. Roberts \& R.L Victoria (eds.), Amazonian Deforestation and Climate. Wiley, Chichester, Reino Unido, 611p.

MAHOWALD, N.M. \& LUO, C. 2003. A less dusty future? Geophysical Research Letters, 30(17): 1903, doi: 10.1029/2003GL017880.

MARENGO, J.A.; NOBRE, C.A.; TOMASELLA, J.;. OYAMA, M.D.; SAMPAIO de OLIVEIRA, G.; de OLIVEIRA, R.; CAMARGO, H.; ALVES, L.M. \& BROWN, I.F.. 2008. The drought of Amazonia in 2005. Journal of Climate, 21: 495-516.

MCPHADEN, M.J.; ZEBIAK, S.E. \& GLANTZ, M.H. 2006. ENSO as an integrating concept in earth science. Science, 314: 1740-1745.

MEEHL, G.A.; STOCKER, T F.; COLLINS, W.D.; FRIEDLINGSTEIN, P.; GAYE, A.T.; GREGORY, J M.; KITOH,
A.; KNUTTI, R.; MURPHY J M.; NODA, A.; RAPER, S.C B.; WATTERSON, I.G. ; WEAVER, A.J. \& ZHAO, Z-C. 2007. Global Climate Projections. p. 247-845 In: S. Solomon, D. Qin, M. Manning, Z. Chen, M. Marquis, K.B. Averyt, M. Tignor \& H.L. Miller, (eds.), Climate Change 2007: The Physical Science Basis. Contribution of Working Group I to the Fourth Assessment Report of the Intergovernmental Panel on Climate Change, Cambridge University Press, Cambridge, Reino Unido, 996p.

NEPSTAD, D.C.; CARVALHO, G.; BARROS, A.C.; ALENCAR, A.; CAPOBIANCO, J.P.; BISHOP, J.; MOUTINHO, P.; LEFEBVRE, P.; SILVA, JR., U.L. \& PRINS, E. 2001. Road paving, fire regime feedbacks, and the future of Amazon forests. Forest Ecology and Management, 154: 395-407.

NEPSTAD, D.C.; LEFEBVRE, P.; SILVAJr., U.L; TOMASELLA, J.; SCHLESINGER, P.; SOLORZANO, L.; MOUTINHO, P.; RAY, D. \& BENITO, J.G. 2004. Amazon drought and its implications for forest flammability and tree growth: A basinwide analysis. Global Change Biology, 10(5): 704-712.

NEPSTAD , D.C.; SOARES-FILHO, B.; MERRY, F.; MOUTINHO, P.; RODRIGUES, H.O.; BOWMAN, M.; SCHWARTZMAN, S.; ALMEIDA, O. \& RIVERO, S. 2007. The Costs and Benefits of Reducing Carbon Emissions from Deforestation and Forest Degradation in the Brazilian Amazon. Woods Hole Research Center (WHRC), Falmouth, Massachusetts, E.U.A. 26p.

NICHOLLS, N. \& 98 outros. 1996. Observed climate variability and change. p. 133-192 In: J.T. Houghton, L.G. Meira Filho, B.A. Callander, N. Harris, A. Kattenberg \& K. Maskell, (eds.), Climate Change 1995: The Science of Climate Change. Cambridge University Press, Cambridge, Reino Unido, 572p.

O ESTADO DE SÃO PAULO, 2009. “Amazônia pede a Lula nova política para floresta”, 01 de julho de 2009, p. A-20.

PHILLIPS, O.L. \& 65 outros. 2009. Drought sensitivity of the Amazon rainforest. Science, 323: 1344-1347.

SALAZAR, L.F.; NOBRE, C.A. \& OYAMA, M.D. 2007. Climate change consequences on the biome distribution in tropical South America. Geophysical Research Letters, 34: L09708, doi: 10.1029/2007GL029695.

SAMPAIO, G.; NOBRE, C.A.; COSTA, M.H.; SATYAMURTY, P.; SOARES-FILHO, B.S. \& CARDOSO, M. 2007. Regional climate change over eastern Amazonia caused by pasture and soybean cropland expansion. Geophysical Research Letters, 34: L17709, doi:10.1029/2007GL030612. 
TRENBERTH, K.E. \& SHEA, D.J. 2006. Atlantic hurricanes and natural variability in 2005. Geophysical Research Letters, 33: L12704, doi: 10.1029/2006GL026894.

VASCONCELOS, S.S. \& BROWN, I.F. 2007. The use of hot pixels as an indicator of fires in the MAP region: Tendencies in recent years in Acre, Brazil. p. 4549-4556. In: J.C.N. Epiphanio, L.S. Galvão \& L.M.G. Fonseca, (eds.), Anais XIII Simpósio Brasileiro de Sensoriamento Remoto, Florianópolis, Brasil 2126 abril 2007. Instituto Nacional de Pesquisas Espaciais (INPE), São José dos Campos, São Paulo, Brasil.

UN-FCCC (United Nations Framework Convention on Climate Change). 1992. United Nations Framework Convention on Climate Change. (disponível em inglês em http://www.unfccc.de e em Português em http://www.mct.gov.br/clima).

Submetido em 15/09/2009.

Aceito em 09/11/2009. 